\title{
Proposal for Modifications to the Bangkok Urban Health System that would Improve the Quality of Health, Independent Living, and Maintenance of Older Adults with Fall-related Trauma (Bangkok Falls
}

\section{Study)}

Jiraporn Sri-on ( $\sim$ Jiraporn.rew@gmail.com )

Navamindradhiraj University

Thiti Kredarunsooksree

Ratchaphiphat Hospital

Thitiwan Paksophis

Navamindradhiraj University

Khemika Rojtangkom

Navamindradhiraj University

Rapeeporn Rojsaengroeng

Navamindradhiraj University

Alissara Vanichkulbodee

Navamindradhiraj University

Yupadee Fusakul

Navamindradhiraj University

Rasida Ruangsiri

Thai Health Promotion Organization (ThaiHealth)

\section{Research Article}

Keywords: Bangkok urban health system, quality of health, independent living, trauma

Posted Date: August 26th, 2021

DOI: https://doi.org/10.21203/rs.3.rs-428302/v1

License: (c) (i) This work is licensed under a Creative Commons Attribution 4.0 International License.

Read Full License 


\section{Abstract}

\section{Background}

The Bangkok falls study aimed to identify fall-associated factors, including home healthcare hazards, nutritional status, hydration status, sarcopenia, frailty, locomotive syndrome, and health status of urban older adults in a middle-income country.

\section{Methods}

This was a population-based cohort study that enrolled adults who lived in Bangkok, Thailand. Our study recruited older adults aged $\geq 60$ years old, able to walk, and expected to live in the community for at least 2 years. The study had three phases included; phase 1: subject identification and terminology clarification. Phase 2: we collected data at community sites on baseline characteristic and fall risk identification. Examinations and laboratory investigations were scheduled for one month later. Phase 3: telephone follow up for falls rate, functional status and death at $3,6,12$ months.

\section{Results}

A total 1,001(51.84\%) people were enrolled for our study. The average age of our study was 69.9 years old (SD, 6.8), and two-thirds were female. Using "Stopping Elderly Accidents, Death and Injuries" (STEADI) screening fall risk, our study found that $37.7 \%$ had scores $\geq 4$, which means that there is a risk of fall. In addition, the risk of falls increased among older adults aged $75-84$ years $(49.5 \%)$ and older adults aged $\geq 85$ years $(67.7 \%)(P$-value $<0.001)$.

\section{Conclusion}

This study demonstrated the feasibility of conducting a population-based cohort study among urban older adults in a middle-income country using the local community healthcare system. Our study have a tendency to provide data source for fall risk factors and disability in older adults.

\section{Introduction}

Falls and fall-related injuries are a leading cause of death among older adults and thus have become a growing public health concern worldwide [1,2]. Injuries from falls often cause a decline in the activities of daily living and can lead to post-fall syndrome, such as immobilization, hip fracture, head injury, and death [3-5]. Falls can happen anywhere, at any time; therefore, fall prevention measures should be taken. A fall risk assessment considers factors such as environmental conditions, functional status, and underlying diseases [6-11]. Fall risk factors have been studied for many decades; however, the causes of falls are so varied that a fall risk assessment will not account for many unknown variables that can arise [6-11]. In addition, the fall risk assessment tools that are commonly used by older adults provide moderate predictive ability for differentiating between high risk and low-risk falls $[6,12,13]$. Because falls are a main cause of disability in older adults and factors that contribute to falls are risk factors for 
numerous adverse events, a fall prevention plan may reduce the risk of disability in older adults. The "MOBILIZE Boston study" evaluated the impacts of pain [14-18], cerebral perfusion, and foot disorder on fall risks among US community older adults. Because certain risk factors for falls, including sarcopenia [19-22], locomotive syndrome (LS) [23, 24] hydration status [25, 26], and nutrition status [27, 28], are not well understood among Southeast Asian populations, large observational studies are required. An assessment for sarcopenia is usually not included among fall risk screening tools, although it is an important predictor for falls and fractures [19-22].

Identifying health conditions associated with falls is imperative for establishing prevention measures. Hypertonic dehydration is associated with falls $[25,26]$, as dehydration causes decreased brain perfusion and orthostatic hypotension. Malnutrition is associated with inhospital falls [27]. A three-year longitudinal study showed an increased risk among aging Taiwanese adults with malnutrition [29], whereas a study in Australia contrarily found no association between falls and malnutrition over a one-year period in community-dwelling older adults [28].

The Bangkok falls study is a population-based cohort study that is part of the Geriatric Emergency Department Research Unit, Rehabilitation, Home Health Care Unit and Vajira Research Facilitation Division, Navamindradhiraj University program project, in collaboration with the Thai Health Promotion Foundation. Each of the studies within the Bangkok falls study aimed to identify fall-associated factors, including home healthcare hazards, nutritional status, hydration status, sarcopenia, frailty, LS, and health status of urban older adults in a middle-income country.

\section{Methods}

A large population of urban older adults were recruited and assessed for individual characteristics, which were stored in a database for cross-study research on the correlation between sarcopenia, LS, and biomarkers and the risk for falls and immobility in older populations. The project was funded by Thai Health Promotion and approved by the Vajira Institutional Review Board.

\section{Study design}

This was a population-based cohort study that enrolled adults age 60 years or older who lived in one of five subdistricts of the Dusit District, Bangkok, Thailand, between October 1, 2019, and September 30, 2021.

The present study had three phases of data collection:

Phase 1: This phase included subject identification and terminology clarification. Experts from Thai Health Promotion, Navamindradhiraj University, Thai College of Emergency Physician, Medical Service Department of Bangkok (20 participants), and the Head and Member of Community Health Promotion Volunteers for each subdistrict (46 participants) met to clarify the association between falls and health 
status. Research assistants (RAs) obtained data on older adults aged $\geq 60$ years who resided in the area and were willing to participate. In Bangkok, health promotion volunteers are employed by the Bangkok Metropolitan Council to work on health promotion at the subdistrict level. They conduct surveys on health status and family members and report data to the Bangkok Department of Health. RAs coordinated the schedule.

Phase 2: At each community site, the principal investigator ( $\mathrm{PI}$ ) and RAs collected data on baseline characteristic and distributed fall calendar postcards and fall risk identification cards reporting the "Stopping Elderly Accidents, Death and Injuries" (STEADI) [30]. Examinations and laboratory investigations were scheduled for one month later.

Phase 3: RAs performed telephone follow-up at 3, 6, and 12 months, which is the gold standard protocol for falls. Respondents answered questionnaires about the activities of daily living, history of falls, rating of the emergency department, hospitalization, and mortality. The results were submitted to the Head of Community and the Department of Health to generate a screening tool and health system for reducing fall occurrence. The subgroups of the cohort were invited to address questions related to the mechanism and prevention of falls, including the correlation between falls and muscle mass and sarcopenia based on ultrasound muscle. A second project determined the prevalence of dehydration and developed a new protocol for older adults.

\section{Recruitment process}

The Head of Community Health Promotion Volunteer announced the project to each subdistrict and recruited older adults who were interested in participating and met the following criteria: aged $\geq 60$ years old, able to walk, and expected to live in the community for at least 2 years. When there were at least 30 participants in a subdistrict, RAs conducted a screening at the community meeting place to determine eligibility. The inclusion criteria were adults $\geq 60$ years old who lived in Dusit District, could walk at least 6 meters, and were expected to live in the community for at least 2 years. The exclusion criteria were older adults who were unable to speak Thai, had severe cognitive impairment of $>12$ points on the sixitem cognitive screening test [31], or were blind or deaf.

\section{Data collection (Table 1 and Figure 1)}

Data on baseline characteristics, underlying diseases, medications, Charlson comorbidity index [32], Barthel activities of daily living index [33], Mini Nutritional Assessment (MNA) [34], cognitive function, World Health Organization Quality of Life (WHOQOL-BREF-THAI) [35], frailty phenotype [36], mobility, fall history, and end of life decision were collected for each participant. RAs obtained written informed consent from each participant. Participants received \$8 USD for each community visits and \$15 USD for the hospital appointment. Transportation was provided based upon request. Details of data collection methods are shown in Table 1 and Figure 1. 


\section{Table 1}

\section{Data collection}

\begin{tabular}{|ll|}
\hline Domain & Community site interview \\
\hline Falls & Sociodemographic information \\
& $\begin{array}{l}\text { History of falls, fracture, and injuries } \\
\text { Fall risk medications }\end{array}$ \\
& Home environment \\
\hline Frailty & Frailty phenotype [36] \\
\hline Footwear & Footwear/shoe wear \\
\hline Nutrition & MNA \\
\hline Medications & Medication inventory (prescription, over-the-counter drug, herbal, or supplemental) \\
\hline Comorbidity & Charlson comorbidity index [32] \\
\hline Cognition & Six-item cognitive screening test [31] \\
\hline Quality of life & WHOQOL-BREF-THAl [35] \\
\hline Behaviors & Alcohol use, smoking, water or other drink \\
\hline Pain & Pain location and characteristics \\
\hline Disability & Barthel activities of daily living index, LS checklist [37] \\
\hline
\end{tabular}

\section{Community site interview}

Five trained RAs performed extensive interviews at the community sites and evaluated health status based on Charlson comorbidity index (self-reported physician diagnosis and confirmation by hospital medical records), sociodemographic information (age, gender, marital status, profession, monthly income, caregiver, family member, level of education, and electronic device for communication), disability (Barthel activities of daily living index), cognitive test (six-item cognitive screening test), and quality of life (WHOQOL-BREF-THAI).

\section{Nutritional assessment [34]}

Nutritional assessment was based on weight, height, body mass index, mid-arm circumference, and the MNA. The MNA score in the elderly can determine adequate nutritional status (MNA, $\geq 24$ ), malnutrition 
risk (MNA, 17-23.5), and protein-calorie malnutrition (MNA, < 17). The MNA had high sensitivity (96\%) and specificity (98\%) for identifying patients who were at risk for malnutrition (MNA, 17-23.5).

\section{Frailty [36]}

Frailty was evaluated using the "frailty phenotype," [36] which has a set of five criteria: involuntary weight loss of $4.5 \mathrm{~kg}$ over the past year, self-reported exhaustion, slow gait speed, poor handgrip strength, and low physical activity. The results were classified as follows: not frail, no criteria present; pre-frail, one or two criteria present; and frail, three or more criteria present.

\section{Medications}

A medication review was performed at a community visit. RAs examined all containers for prescription, over-the-counter, and herbal medicines used in the previous month, and the name, daily dose, and strength were recorded. Upon hospital examination, PI confirmed the RAs' record of medication used with the hospital electronic medical record system.

\section{Fall assessment}

Fall evaluations were determined based on the STEADI guideline and geriatric emergency department guideline. The home environment was assessed based on a checklist to assess for fall hazards such as imbalanced stair, improper lighting in the hallway, hand drill in the bathroom, and slippery floor.

\section{Quality of life [35]}

WHOQOL-BREF-THAI was used to evaluate the individual's perception of participants in the main concept of their goal, expectations, and value of life. It has 26 items assessed on a scale of $0-5$. Therefore, the final score can range from 0 to 130 . Four domains are evaluated: physical health, psychological wellbeing, social relationships, and the environment. Our study modified the score for each domain to $0-100$, following the WHO recommendations. A higher score indicates better perception of quality of life.

\section{Clinical examination}

Evaluation for clinical examination was performed by three emergency physicians who had experienced taking care of older adults for at least 5 years, three RAs who had a bachelor's degree in health science, and experienced nurse practitioners. Before the examination started, one physiatrist trained all physicians and RAs to evaluate their physical performance. The examiner was carefully evaluated and gave participants 5-10 minutes of rest before each step of evaluation to avoid overexhaustion. 


\section{Mobility performance, muscle strength, and musculoskeletal examination}

The purpose of examination was to assess muscle strength and muscle power, especially of the proximal muscle. The mobility performance assessment during hospital visit included time taken to get up and go and the 4-meter test. LS was established and defined by the Japanese Orthopedic Association in 2007 as a state of degraded mobility due to impaired locomotive organs, which increases the risk of disability. Decreased motor function and musculoskeletal pathologies include degenerative spondylosis and spinal stenosis. LS in this study was defined using a geriatric locomotive function scale of 25 questions (GLFS25) [37]. A score of $\geq 16$ indicates LS. The study showed that GLFS-25 is associated with decreased gait speed and a risk of falls $[13,14]$.

\section{Fall evaluations}

During hospital visits, RAs recorded orthostatic vital signs, the time taken to get up and go [39-41], physical performance [38,42-45], somatosensory function, and ultrasound muscle mass.

Each participant received a telephone follow-up at 3, 6, and 12 months.

\section{Physical performance [42-45]}

The lower extremity mobility performance was evaluated using the Short Physical Performance Battery (SPPB) [38]. The SPPB measures the total time taken to perform the sit to stand test five times, 4-meter walking speed [41], and balance while standing. The full range was based on a scale from 0 to 12 .

\section{Berg balance scale}

The Berg Balance Scale consists of 14 balance tasks. The scale has been validated as a fall risk assessment tool for community-dwelling older adults.

\section{Somatosensory function test}

Light touch was evaluated using Semmes-Weinstein monofilament test (size, 5.07; weight, $10 \mathrm{~g}$ ). We tested the greater toe and the head of the first, third, and fifth metatarsal areas. The test results were categorized as sensory deficit and no deficit.

\section{Sarcopenia [46]}


Sarcopenia was defined as an age-related loss of skeletal muscle, decreased muscle strength, and low physical performance. For identifying patients at risk for sarcopenia, we followed the Asian Working Group for Sarcopenia 2019 criteria for diagnosis.

Criteria for sarcopenia diagnosis

Grip strength was evaluated using the grip dynamometer model TK-1201 (TAKEI KIKI KOGYO, Japan) (male, $<28 \mathrm{~kg}$; female, $<18 \mathrm{~kg}$ ). Physical performance was evaluated based on a 6-meter walk $(<1.0$ $\mathrm{m} / \mathrm{s}$ ) or 5 -time chair stand test ( $\geq 12$ seconds). Appendicular skeletal muscle mass (ASM) was evaluated using BIA (male, $<7.0 \mathrm{~kg} / \mathrm{m}^{2}$; female, $<5.7 \mathrm{~kg} / \mathrm{m}^{2}$ ). Sarcopenia was defined as having low ASM plus low muscle strength or low physical performance. Severe sarcopenia were defined as having low ASM plus low muscle strength and low physical performance.

\section{Dehydration [25]}

We performed a blood test to test the hydration status. Hypertonic dehydration was defined by serum osmolality: current dehydration (>300 mOsm/ $/ \mathrm{kg}$ ), impending dehydration $(295-300 \mathrm{mOsm} / \mathrm{kg}$ ), and normal hydration ( 275 to $<295 \mathrm{mOsm} / \mathrm{kg}$ ).

\section{Statistical analysis}

Statistical analysis was performed using STATA version 15.0. A descriptive statistic was used for the baseline demographic data. We implemented a survival analysis as part of a prospective analysis to assess the risk of falls associated with the risk of sarcopenia and dehydration (not presented in this paper). It is beyond the scope of this study to present all plan analyses performed for the Bangkok falls study; therefore, we have described a standard methodology that will be used as part of our research. The incident of falls will be identified using a new case of a fall with a recurrent fall, which has been presented as a cumulative rate. We analyzed the time to first fall using a survival analysis. Multivariate analysis was used to estimate the relative risk of falls. We used a general linear model to depict repeatedly ordinal outcomes with correlated responses, such as Poisson regression. Other analyses included structural equation modeling to determine whether there are latent variables, as defined by share covariate potential fall risk factors.

\section{Recruitment}

The recruitment and enrollment process is depicted in Figure 2. Our study identified 1,931 (11.2\%) older adults aged $\geq 60$ from the total population $(N=17,228)$ from Dusit District.

A total of 1,107 people were screened, of which 824 (42.76\%) refused to participate in the study. After the screening process, the following information were obtained: 91 (4.7\%) people were bedridden, $10(0.52 \%)$ 
people scored more than 12 on the six-item cognitive screening, and $5(0.26 \%)$ people were blind or deaf. Finally, 1,001(51.84\%) people were eligible for our study.

\section{Baseline characteristics}

The average age of our study was 69.9 years old (SD, 6.8), and two-thirds were female. Of the study cohort, $100 \%$ were Asian, and $98.40 \%$ were Buddhist. The study cohort had a high prevalence for education less than high school (57.44\%) and unemployment (51.65\%). The rate of unemployment increased among older adults aged $\geq 85$ years old. Most of the participants had a caregiver $(78.4 \%)$. The average age of the caregiver increased among older adults aged $\geq 85$ years old (mean, $58.6 \pm 15.4$ years). Hypertension was the most common underlying disease, and the rate increased with age. Data are shown in table 2.

Table 2

Baseline characteristics

\section{Functional status, fall history, and quality of life (Table 3)}

Almost half of the older adults in our cohort had mild to moderate cognitive impairment. One-third had a frailty phenotype score of $\geq 3$. Our study revealed that $16.4 \%$ were obese, and one-fifth (18.6\%) were at high risk for malnutrition based on the MNA. Overall, $25 \%$ had experienced falls within 1 year, and the rate increased among older adults aged $\geq 85$ years old.

Using STEADI screening fall risk, our study found that $37.7 \%$ had scores $\geq 4$, which means that there is a risk of fall. In addition, the risk of falls increased among older adults aged $75-84$ years (49.5\%) and older adults aged $\geq 85$ years $(67.7 \%)(P$-value $<0.001)($ Table 3$)$.

Table 3

Functional status, fall history, and quality of life 


\begin{tabular}{|c|c|c|c|c|c|}
\hline \multirow[t]{3}{*}{ Characteristic } & \multirow{3}{*}{$\begin{array}{l}\text { Total } \\
N=1,001(\%)\end{array}$} & \multicolumn{4}{|l|}{ Age (year) } \\
\hline & & $60-74$ & $75-84$ & 85 & P- \\
\hline & & $\mathrm{N}=764(\%)$ & $\mathrm{N}=206(\%)$ & $\mathrm{N}=31(\%)$ & \\
\hline \multicolumn{6}{|l|}{ Gender } \\
\hline Female & $690(68.9)$ & $529(69.2)$ & $141(68.5)$ & $20(64.5)$ & 0.844 \\
\hline Age (year), mean (SD) & $69.9(6.82)$ & $66.8(2.7)$ & $78.6(2.7)$ & $86.9(2.0)$ & \\
\hline Age (year), median(IQR) & $69(64,74)$ & $67(63,70)$ & $78(76,80)$ & $87(85,88)$ & \\
\hline Religion & & & & & 0.446 \\
\hline Buddhism & 985(98.4) & 752(98.4) & 203(98.5) & $30(96.8)$ & \\
\hline Christianity & $5(0.5)$ & $3(0.4)$ & $1(0.5)$ & $1(3.2)$ & \\
\hline Islam & $10(1.0)$ & $8(1.1)$ & $2(1.0)$ & 0 & \\
\hline Sikhism & $1(0.1)$ & $1(0.1)$ & 0 & 0 & \\
\hline Education level & & & & & 0.001 \\
\hline Uneducated & $77(7.7)$ & $48(6.3)$ & $22(10.7)$ & $7(22.6)$ & \\
\hline Less than high school & $575(57.4)$ & $437(57.2)$ & $121(58.7)$ & $17(54.8)$ & \\
\hline High school & $302(30.3)$ & 239(31.3) & $59(28.6)$ & $4(12.9)$ & \\
\hline College or higher & $47(4.6)$ & $40(5.2)$ & $4(1.9)$ & $3(9.7)$ & \\
\hline Profession & & & & & 0.006 \\
\hline Unemployee & $517(51.7)$ & $370(48.4)$ & $125(60.7)$ & $22(71.0)$ & \\
\hline General trading career & 182(18.2) & 144(18.9) & $36(17.5)$ & $2(6.5)$ & \\
\hline Employment & 155(15.5) & $136(17.8)$ & $16(7.8)$ & $3(9.7)$ & \\
\hline Others & $95(9.5)$ & 73(9.6) & $20(9.7)$ & $2(6.5)$ & \\
\hline Retired government employee & $52(5.2)$ & $41(5.4)$ & $9(4.4)$ & $2(6.5)$ & \\
\hline Has care giver & 785(78.4) & $591(77.4)$ & 171(83.0) & $23(74.2)$ & 0.173 \\
\hline Age of care giver (year), mean (SD) & $\begin{array}{l}52.5 \\
(17.92)\end{array}$ & $50.9(16.9)$ & $57.0(18.1)$ & $58.6(15.4)$ & $<0.001$ \\
\hline Caregiver & & & & & 0.147 \\
\hline Secondary-degree relative & $140(14.0)$ & 103(17.4) & $33(19.2)$ & $4(17.4)$ & \\
\hline First-degree relative & $346(34.6)$ & $251(42.5)$ & $80(46.5)$ & $15(65.2)$ & \\
\hline Spouse & $273(21.5)$ & 218(36.9) & $52(30.2)$ & $3(13.0)$ & \\
\hline
\end{tabular}




\begin{tabular}{|llllll|}
\multicolumn{1}{|c}{ Others } & $27(2.7)$ & $19(3.2)$ & $7(4.1)$ & $1(4.4)$ & \\
$\begin{array}{l}\text { Charlson co-morbidity score, mean } \\
\text { (SD) }\end{array}$ & $3.24(1.3)$ & $2.9(1.1)$ & $4.3(1.2)$ & $4.7(0.7)$ & $<0.001$ \\
$\quad$ Hypertension & & & & & \\
\multicolumn{1}{c}{ Dyslipidemia } & $585(58.4)$ & $425(55.6)$ & $138(67.0)$ & $22(71.0)$ & 0.005 \\
& $428(42.8)$ & $318(41.6)$ & $95(46.1)$ & $15(48.4)$ & 0.416 \\
Alcohol & $86(8.6)$ & $78(10.2)$ & $6(2.9)$ & $2(6.5)$ & 0.001 \\
\hline Smoking & $85(8.5)$ & $78(10.2)$ & $7(3.4)$ & 0 & 0.001 \\
\hline
\end{tabular}




\begin{tabular}{|c|c|c|c|c|c|}
\hline \multirow[t]{2}{*}{ Characteristic } & \multirow{2}{*}{$\begin{array}{l}\text { Total } \\
\mathrm{N}=1,001(\%)\end{array}$} & \multicolumn{4}{|l|}{ Age (year) } \\
\hline & & $60-74$ & $75-84$ & 85 & $\begin{array}{l}\mathrm{P} \\
\text { value }\end{array}$ \\
\hline $\mathrm{BMI}\left(\mathrm{kg} / \mathrm{m}^{2}\right)$, mean (SD) & $25.8(4.7)$ & $26.2(4.9)$ & 24.7(3.7) & $24.5(4.9)$ & \\
\hline$<18.5$ & $49(4.7)$ & $39(5.1)$ & $7(3.4)$ & $3(9.7)$ & $<0.001$ \\
\hline $18.5-29.9$ & 788(78.7) & $582(76.2)$ & 185(89.8) & $21(67.7)$ & \\
\hline 30 & 164(16.4) & 143(18.7) & $14(8.8)$ & $7(22.6)$ & \\
\hline 6-CIT score & & & & & $<0.001$ \\
\hline $0-7$ & $539(53.9)$ & 442(57.9) & $85(41.3)$ & 12(38.7) & \\
\hline $8-9$ & $324(32.4)$ & 238(31.2) & 79(38.4) & $7(22.6)$ & \\
\hline $10-12$ & 138(13.8) & $84(11.0)$ & $42(20.4)$ & 12(38.7) & \\
\hline Use of walking aid & $91(9.1)$ & $44(5.8)$ & $36(17.5)$ & $11(35.5)$ & $<0.001$ \\
\hline Frailty score (3) & $307(30.7)$ & $215(28.1)$ & $80(38.8)$ & 12(38.7) & 0.008 \\
\hline History of fall in 1 year & $252(25.2)$ & $190(24.9)$ & $52(25.2)$ & $10(32.3)$ & 0.626 \\
\hline Indoor falls & $124(49.2)$ & $92(48.4)$ & $25(48.1)$ & $7(70.0)$ & 0.457 \\
\hline Outdoor falls & $128(50.8)$ & $98(51.6)$ & $27(51.9)$ & $3(30.0)$ & \\
\hline STEADI score (4) & $377(37.7)$ & 254(33.3) & $102(49.5)$ & $21(67.7)$ & $<0.001$ \\
\hline Eye examination within 1 year & 498(49.8) & $362(47.4)$ & 115(55.8) & 21(67.7) & 0.012 \\
\hline Mini Nutrition Assessment Score & & & & & 0.001 \\
\hline $24-30 \quad:$ Normal nutritional & $804(80.3)$ & 633(82.9) & 152(73.8) & 19(61.3) & \\
\hline $\begin{array}{l}17-23.5 \text { : High risk for } \\
\text { malnutrition }\end{array}$ & 186(18.6) & 125(16.4) & $50(24.3)$ & $11(35.5)$ & \\
\hline$<17$ : Malnourished & & & & & \\
\hline WHO QOL-BREF-THAI & & & & & \\
\hline Domains & & & & & \\
\hline 1. Physical health & $48.9(9.8)$ & $49.0(11.1)$ & 48.8(11.7) & $46.2(9.8)$ & 0.361 \\
\hline 2. Psychological & $57.5(12.4)$ & $57.5(12.4)$ & $57.4(12.5)$ & $58(10.9)$ & 0.968 \\
\hline 3. Social relationships & $60.5(17.8)$ & $60.7(17.4)$ & $60.2(18.9)$ & $58.7(19.4)$ & 0.789 \\
\hline 4. Environment & 70.3(14.1) & 70.1(14.0) & 70.7(14.6) & $70.8(14.2)$ & 0.837 \\
\hline
\end{tabular}




\section{Data collection}

From a total of 13 community visits, the median number of participants was 86 (IQR, 52-94). The estimate average length of the interview was 20 minutes per person. One month after the community visits, we scheduled each participant for clinical examination at the hospital. Of the participants, five had died, four refused to visit the hospital, and 90 could not be contacted because of the COVID-19 lockdown policy implemented in Bangkok, which forced the relocation of some older adults to rural areas; they were no longer part of the community population.

A total of $902(90.1 \%)$ participants completed the hospital follow-up visits. The median length of clinical examination was 2.2 hours (IQR, 1.5-3.0) per person. Of the 902 participants, 898 (99.5\%) performed the timed up and go, 4-meter walk test, and SPPB test. All participants had ultrasound muscle mass and inferior vena cava vein. There were four participants for which the blood test results could not be interpreted. No participants reported adverse events associated with the research. At the 12-month follow-up visit, 832 participants were retained in the study. Six participants died (data not presented).

\section{Discussion}

This study demonstrated the feasibility of a population-based study of community-dwelling older adults aged $\geq 60$ years residing in a middle-income country to identify fall risk factors with the aim of developing a community health system that can reduce the number of events of falls. The results showed excellent information obtained from community visits, as there was no missing data. For the hospital visit, the data obtained from participants was good; however, the COVID-19 outbreak and the lockdown policy prevented contact with some of the participants (90/1001 (9\%)). However, 99\% participants who visited the hospital completed leg examination, ultrasound for the evaluation of muscle mass, and blood tests for the hydration status. The rate of completed leg examination was higher than the MBS [15] study because we included older adults aged $\geq 60$ years, which was younger than the ages of participants included in the MBS study (adults aged > 70 years). The older the age range of participants, the higher the percentage of reported frailty and use of walking aids.

Participants reported a positive experience from their participation in the study, as the rate of retention was $\sim 90 \%$. Our research team took precautions regarding the comfort and safety of the participants. At the community visits, we provided breaks with the option for snack or lunch and rest periods to reduce fatigue and exhaustion. We had a Line group connection with participants who were willing to receive information or health recommendations about falls from our research team (Line is a freeware app for instant communication via electronic devices such as smartphones, tablets, or computers) [47].

Our study evaluated fall risk based on a combination of environmental causes, cognitive and physical function, balance and physical performance, hydration and nutritional status, and sarcopenia. Several studies have described reasons for falls, including gait and balance limitations, that have not been well defined, such as weakness or loss of stability $[1,6,7]$. The roles of sarcopenia, hydration status, and 
nutritional status have not yet been explored in other fall studies. Furthermore, studies on the use of rapid screening tools, such as ultrasound screening for sarcopenia, and their efficacy in evaluating fall risk in older adults have been limited. The Bangkok falls study provides a resource for future research, especially in Asian countries where cultural and environmental influences have been reported as factors in fall risk.

Using a population-based study allowed us to generalize our results in comparison with other populations of older adults. Our study cohort represented Asian older adults without significant severe cognitive impairment who live in an urban community setting and are able to walk around their home. Thus, 42\% refused to participate in our study for the primary reason that they did not have time to participate in the community site visits on the weekend for various reasons such as having a trade or job commitment on the weekend. In Bangkok, people generally work on weekends, especially when they are a trader.

Because of the limitations of the baseline population-based study, we set the study aims for five projects. The fall risks were central and may not have been specific to each project. We selected only a few factors to use as adjustment variables for performing the multivariate analyses rather than a measurement of primary exposure. For example, we determined the peripheral neuropathy of the foot rather than specific deformities, such as narrow toe boxes, absent fixation, or excessively flexible heel counter or soles.

\section{Conclusion}

This study demonstrated the feasibility of conducting a population-based cohort study among urban older adults in a middle-income country using the local community healthcare system. Our results explored fall risk factors and disability in older adults. The study revealed that older adults in urban areas feel energetic interest in performing an activity and a desire to participate in this type of research. The Bangkok falls study supplies a data resource for fall risk factors in urban older adults.

\section{Abbreviations}

LS: locomotive syndrome, RAs: Research assistants, STEADI: Stopping Elderly Accidents, Death and Injuries, MNA: Mini Nutritional Assessment, WHOQOL-BREF-THAI: World Health Organization Quality of Life, ASM: Appendicular skeletal muscle mass, SD: Standard deviation, SPPB: the Short Physical Performance Battery, IQR : Interquartile range

\section{Declarations}

\section{Ethics approval and consent to participate}

Our study was approved by Vajira institutional review board which adheres to the Declaration of Helsinski, the Belmont Report, CIOMS Guideline, ICH-GCP. (Ref: COA 107/2562). The informed consent was obtained from all subjects involved in the study and legally authorized representatives of dead patients. 


\section{Consent for publication}

Not applicable

\section{Availability of data and materials}

All data generated and/or analysed during the current study available from corresponding author on reasonable request.

\section{Competing interests}

The authors have no potential conflicts of interest to disclose.

\section{Funding}

Thai Health Promotion Foundation

\section{Authors' contributions}

JS, TK, TP, YF, RRu conceived and design a study. JS, TP, KR , AV, RRo and RRu acquisition of the data. JS, TK and TP analyses and interpretation of the data. JS and TP drafted of the manuscript. JS, RRu, RRa, YF critical revision of the manuscript for important intellectual content and statistical expertise. All authors approved the final version of the manuscript to be published

\section{Acknowledgments}

The authors would like to thank Thai Health Promotion Foundation for funding this study and Vajira Hospital for allowing this study to commence.

\section{References}

1. TinettiME,SpeechleyM,GinterSF.Risk factors for falls among elderly persons living in the community.N Engl J Med1988;319:1701-7.

2. HartholtKA,LeeR,BurnsER,vanBeeckEF.Increase in fall-related hospitalization in the United States, 2001-2008.J Trauma.2011;71:255-8.

3. Hartholt KA, Lee R, Burns ER, Beeck EV. Mortality from falls among US adults aged 75 years or older, 2000-2016. JAMA 2019;321(21):2131. DOI:10.1001/jama.2019.4185.

4. Koso RE, Sheets C, Richardson WJ, Galanos A. Am J Hosp Palliat Care 2018;35(4):612-9. 
5. Scandol JP, Toson B, Close JCT. Fall-related hip fracture hospitalisations and the prevalence of dementia within older people in New South Wales, Australia: an analysis of linked data. Injury 2013;44(6):776-83.

6. Perrell KL, Nelson A, Goldman RL, Luther SL, Prieto-Lewis N, Rubenstein R. Fall risk assessment measures: an analytic review. J Gerontology A Biol Med Sci 56: M761-6.

7. RubensteinLZ.Falls in older people: epidemiology, risk factors and strategies for prevention.Age and Aging2006;35(Suppl 2):ii37-ii41.

8. TerrosoM,RosaN,TorresMA,SimoesR.Physical consequences of falls in the elderly: a literature review from 1995 to 2010.Eur Rev Aging Phys Act2014;11:51-59.

9. WelmerAK,RizzutoD,LaukkaEJ,JohnellK,FratiglioniL.Cognitive and physical function in relation to the risk of injurious falls in older adults: a population-based study.J Gerontol A Biol Sci Med Sci2017;72:669-75.

10. GaleCR,WestburyLD,CooperC,DennisonEM.Risk factors for incident falls in older men and women: the English longitudinal study of aging.BMC Geriatr2018;18:117.

11. DeandreaS,LucenteforteE,BraviF,FoschiR,LaVecchiaC,NegriE.Risk factors for falls in communitydwelling older people: a systematic review and meta-analysis. Epidemiology 2010;21(5):658-68.

12. Park S. Tool for assessment fall risk in the elderly: a systematic review and meta-analysis. Aging Clin Exp Res 2017;30:1-16.

13. Lee J, Geller Al, Strasser DC. Analytical review: focus on fall screening assessments. PM R;5:609-21.

14. Ogawa EF, Shi L, Bean JF, Hausdorff JM, Dong Z, Manor B, et al.Chronic pain characteristics and gait in older adults: The MOBILIZE Boston Study II.Arch Phys Med Rehabil2020;101(3):418-25.

15. LeveilleSG,KielDP,JonesRN,RomanA,HannanMT,SorondFA,etal.The MOBILIZE Boston Study: Design and methods of a prospective cohort study of novel risk factors for falls in an older population.BMC Geriatr2008;8-16.

16. NawaiA,LeveilleSG,ShmerlingRH,van derLeeuwG,BeanJF.Pain severity and pharmacologic pain management among community-living older adults: the MOBILIZE Boston study. Aging Clin Exp Res 2017;29(6):1139-47.

17. ZhouJ,HabtemariamD,Iloputaifel,LipsitzLA,ManorB.The complexity of standing postural sway associates with future falls in community-dwelling older adults: The MOBILIZE Boston Study. Sci Rep2017;7(1):2924.

18. KangHG,QuachL,LiW,LipsitzLA.Stiffness control of balance during dual task and prospective falls in older adults: the MOBILIZE Boston Study. Gait Posture 2013;38(4):757-63.

19. YeungSS,ReijnierseEM,PhamVK,TrappenburgMC,LimWK,MeskersCG,etal.Sarcopenia and its association with falls and fractures in older adults: A systematic review and meta-analysis.J Cachexia Sacropenia Muscle2019;10(3):485-500.

20. ClynesMA,EdwardsMH,BuehringB,DennisonEM,BinkleyN,CooperC.Definition of sarcopenia: associations with previous falls and fracture in a population sample.Calcif Tissue Int2015;97:445- 
52.

21. GadelhaAB,NeriSGR,deOliveiraRJ,BottaroM,deDavidAc,VainshelboimB,etal.Severity of sacropenia is associated with postural balance and risk of falls in community-dwelling older women.Exp Aging Res2018;44:258-69.

22. HuoYR,SuriyaarachchiP,GomezF,CurcioCL,BoersmaD,MuirSW,etal.Phenotype of osteosarcopenia in older individuals with a history of falling.J Am Med Dir Assoc2015;16:290-5.

23. Ishibashi H. Locomotive syndrome in Japan. Osteoporosis and sarcopenia. 2018; 86-94.

24. Nakamura M, Hashizume H, Oka H, Okada M, Takakura R, Hisari A, et al. Physical performance measures associated with locomotive syndrome in middle-aged and older Japanese women. $J$ Geriatr Phys Ther 2015;38:202-7.

25. Hooper L, Abdelhamid A, Attreed NJ, Campbell WW, Channell AM, Chassagne P, et al. Clinical symptoms, signs and tests for identification of impending and current water-loss dehydration in older people. Cochrane Database Syst Rev. 2015(4):Cd009647.

26. Hamrickl,NortonD,BirstlerJ,ChenG,CruzL,HanrahanL.Association between dehydration and falls.Mayo Clin Proc Innov Qual Outcomes2020;4(3):259-65.

27. EglseerD,HoedIM,SchobererD.Malnutrition risk and hospital-acquired falls in older adults: a crosssectional, multicenter study.Geriatr Gerontol Int2020;20(4):348-53.

28. IsenringE,BakerJ,KerrG.Malnutrition and falls risk in community-dwelling older adults.J Nutr Health Aging2013;17(3):277-9.

29. TsaiAC,LaiMY.Mini nutritional assessment and short-form mini nutritional assessment can predict the future risk of falling in older adults -results of a national cohort study.Clin Nutr2014;33(5):844-9.

30. Center for Disease Control and Prevention.STEADIOlderadultfallprevention..https://www.cdc.gov./steadi/index.html.(AccessedFebruary28,2021)

31. Brooke P, Bullock R. Validation of a 6-item cognitive impairment test with a view to primary care usage. Int J Geriatr Psychiatry 1999;14:936-40.

32. Charlson ME, Pompei P, Ales KL, MacKenzie CR. A new method of classifying prognostic comorbidity in longitudinal studies: development and validation. J Chronic Dis1987;40:373-83.

33. Mahoney FI, Barthel DW. Functional evaluation: the Barthel index. Md Stat Med J. 1965;14:61-5.

34. Vellas B, Guigoz Y, Garry PJ, Nourhashemi F, Bennahum D, Lauque S, Albarede JL. The Mini Nutritional Assessment (MNA) and its use in grading the nutritional state of elderly patients. Nutrition 1999;15(2):116-22.

35. World Health Organization. WHOQOL: measuring quality of life 2012.https://www.who.int/tools/whoqol. (Accessed Feb62021).

36. Fried LP, Tangen CM, Walston J, Newman AB, Hirsch C, Gottdiener J, et al. Frailty in older adults: evidence for a phenotype. J Gerontol A Biol Sci Med Sci 2001;56:M146-56.

37. Kimura A, Takeshita K, Inoue H, Seichi A, Kawasaki Y, Yoshii T, et al. The 25-question Geriatric locomotive function scale predicts the risk of recurrent falls in postoperative patients with cervical 
myelopathy. J Orthop Sci 2018; 23: 185-9.

38. GuralnikJM,SimonsickEM,FerrucciL,GlynnRJ,BerkmanLF,BlazerDG,etal.A Short physical performance battery assessing lower extremity function: association with self-reported disability and prediction of mortality and nursing home admission.J Gerontol1994;49(2):M85-94.

39. PodsiadloD,RichardsonS.Thr timr "Up\&Go": a test of basic functional mobility for frail elderly persons.J Am Geriatr Soc1991;39(2):142-148.

40. ViccaroLJ,PereraS,StudenskiSA.Is timed up and go better than gait speed in predicting health, function, and falls in older adults? J Am Geritr Soc2011;59(5):887-92.

41. BohannonRW,WangYC.Four-meter gait speed: normative values and reliability determined for adults participating in the NIH toolbox study.Arch Phys Med Rehabil2019;100(3):509-13.

42. Berg KO, Maki BE, Williams JI, Holiday PJ, Wood-Dauphinee SL. Clinical and laboratory measures of postural balance in an elderly population. Arch Phys Med Rehabil 1992;73(11):1073-80.

43. Hurvitz EA, Richardson JK, Werner RA, Ruh AM, Dixon MR. Unipedal stance testing as an indicator of fall risk among older outpatients. Arch Phys Med Rehabil 2000;81(5):587-591.

44. The Berg Balance Scale as a clinical screening tool to predict fall risk in older adults: a systematic review.Physiotherapy2018;104(4):383-94.

45. ReiderN,GaulC.Fall risk screening in the elderly: A comparison of the minimal chair height standing ability test and 5-repetition sit-to-stand test.Arch Gerontol Geriatr2016;65:133-9.

46. Chen LK, Woo J, Assantachai P, Auyeung TW, Chou MY, Lijima K, Jang HC, et al. Asian working group for sarcopenia: 2019 consensus update on sarcopenia diagnosis and treatment. JAMDA 2020;300-7.

47. Line.https://line.me/en/(Accessed February28,2021).

\section{Figures}




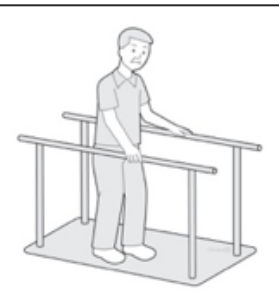

Mobility performance: Short Physical Performance Battery (SPPB) [38], time up and go [40,41], 4-meter normal walking speed [41], test for LS

Balance: Standing balance $+/-$ divided task, Berg balance test [42-44]

Muscle strength: Leg muscle strength and muscle power, five times sit to stand test [45]

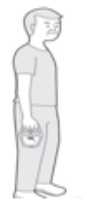

Sarcopenia test [46]: Ultrasound muscle mass and inferior vena cava size, dynamometer, bioelectrical impedance analysis (BIA)

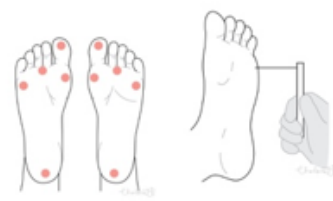

Modified Semmes-Weinstein neuropathy assessment (monofilaments)

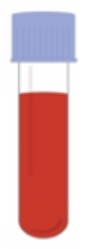

Blood test: Non-fasting blood test, serum osmolarity, electrolyte, blood urea nitrogen, and creatinine

\section{Figure 1}

Clinical Examination 


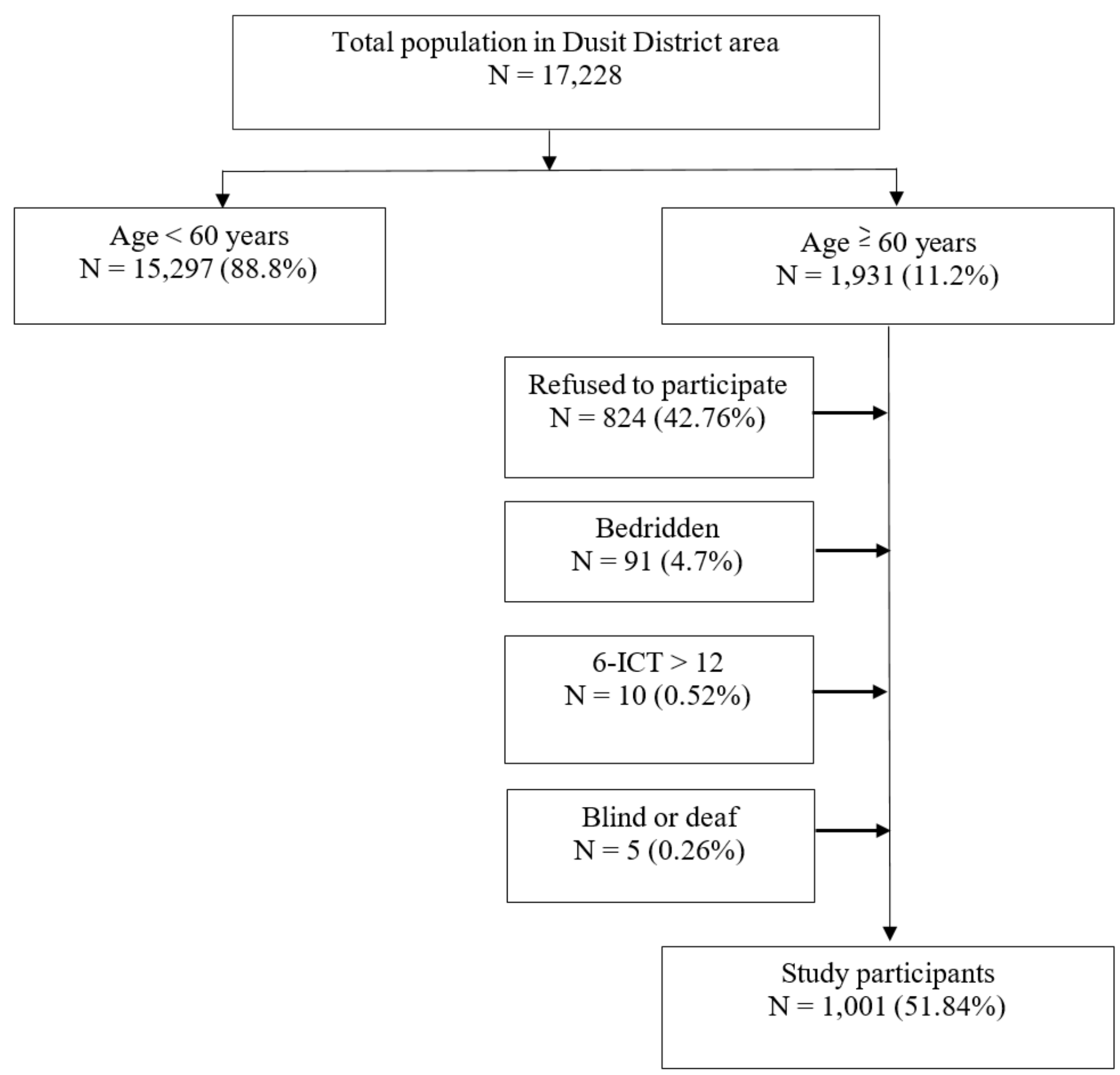

Figure 2

Recruitment and enrollment of participants for the Bangkok fall study 